February 2016

\title{
Pulmonary scedosporiosis mimicking aspergilloma in an immunocompetent host: a case report and review of the literature
}

Fasih Ur Rahman

Aga Khan University

Muhammad Irfan Ul Haq

Aga Khan University

Naima Fasih

Aga Khan University, naima.fasih@aku.edu

Kauser Jabeen

Aga Khan University, kauser.jabeen@aku.edu

Follow this and additional works at: http://ecommons.aku.edu/pakistan_fhs_mc_surg_surg

Part of the Surgery Commons

\section{Recommended Citation}

Rahman, F., Muhammad Irfan Ul Haq, ., Fasih, N., Jabeen, K. (2016). Pulmonary scedosporiosis mimicking aspergilloma in an immunocompetent host: a case report and review of the literature. Infection, 44(1), 127-132.

Available at: http://ecommons.aku.edu/pakistan_fhs_mc_surg_surg/610 


\title{
Pulmonary scedosporiosis mimicking aspergilloma in an immunocompetent host: a case report and review of the literature
}

\author{
Fasih Ur Rahman ${ }^{1}$ Muhammad Irfan ${ }^{1} \cdot$ Naima Fasih $^{2} \cdot$ Kauser Jabeen $^{2} \cdot$ \\ Hasanat Sharif ${ }^{3}$
}

Received: 10 December 2014 / Accepted: 31 August 2015 / Published online: 9 September 2015

(C) Springer-Verlag Berlin Heidelberg 2015

\begin{abstract}
A case of localized lung scedosporiosis is reported here that mimicked aspergilloma in an immunocompetent host. Through this case the importance of considering Scedosporium spp. in differential diagnosis of locally invasive lung infections and fungal ball is highlighted. As it is difficult to differentiate Scedosporium from Aspergillus on clinical grounds, microscopy, radiology and histopathology, this case is further emphasizing the significance of the definitive etiological characterization of Scedosporium through culture or molecular diagnostic tools. Accurate identification of Scedosporium, surgical resection and high-dose voriconazole has been associated with favorable outcome in most reported cases of scedosporiosis.
\end{abstract}

Keywords Scedosporiosis · Lung scedosporiosis · Pulmonary scedosporiosis $\cdot$ Scedosporiosis in an immunocompetent host · Aspergilloma - Treatment of scedosporiosis - Scedosporium apiospermum . Voriconazole

Naima Fasih

fasihnaima@gmail.com

1 Section of Pulmonary and Critical Care Medicine, Department of Medicine, Aga Khan University Hospital, Karachi, Pakistan

2 Department of Pathology and Microbiology, Aga Khan University Hospital, Stadium Road, Karachi 74800, Pakistan

3 Cardio-Thoracic Surgery, Aga Khan University Hospital, Karachi, Pakistan

\section{Introduction}

Scedosporium species is an emergent fungal pathogen associated with a wide range of infections ranging, from subcutaneous mycetoma to disseminated sepsis [1]. Localized invasive lung infection with Scedosporium species, clinically similar to that caused by Aspergillus spp., has been reported in patients with cavitory lung diseases [2, 3]. Treatment of Scedosporium infections is more challenging as it is highly resistant to commonly used antifungal agents especially amphotericin B [1]. Clinical outcome is frequently fatal $(>80 \%)$ especially for disseminated infections [4].

We are reporting here a case of localized lung scedosporiosis in an immunocompetent host with clinical and radiological findings suggestive of aspergilloma. This case emphasizes the importance of considering Scedosporium spp in differential diagnosis of locally invasive lung infections and fungal balls. Culture should be requested for the resected specimen as it is difficult to differentiate Scedosporium from other filamentous molds on clinical grounds, microscopy, radiology and histopathology [2,3].

\section{Case}

A 40-year-old man presented in pulmonology clinic with complaints of recurrent episodes of cough and hemoptysis for the last 4 years. He had a history of pulmonary TB 15 years back. Physical examination revealed bronchial breathing in right upper and middle part of chest. Chest radiograph showed right-sided multiple thick walled cavities of different sizes. Multiple well-defined soft tissue density masses were present in at least two of these cavities. Computed tomography (CT) chest showed two thick-walled 


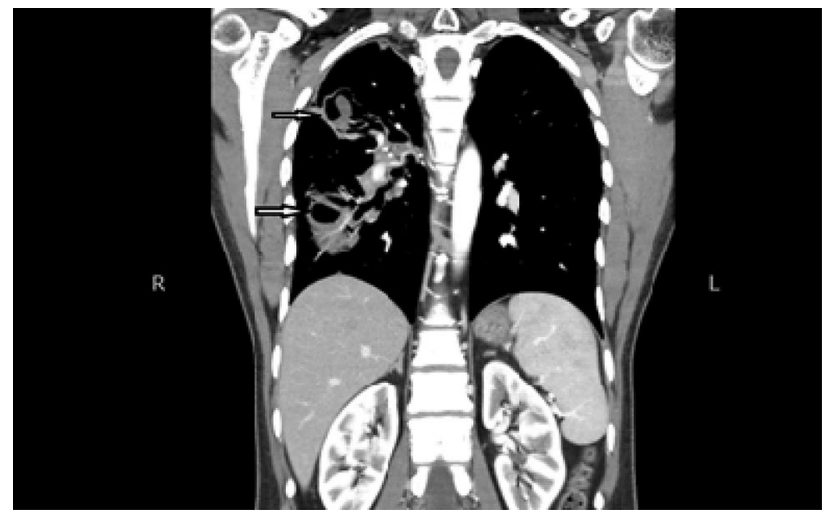

Fig. 1 Computed tomography scan of chest; arrow showing thick walled cavities with air-crescent sign

cavities in right lung. The smaller one was in right upper lobe, $(4.2 \times 2.4 \mathrm{~cm})$ with a fungal ball. The larger cavity $(10 \times 4.5 \mathrm{~cm})$ was in right lower lobe having a large fungal ball $(47 \times 29 \mathrm{~mm})$, extending across major fissure to involve upper lobe as well. Both of these cavities were associated with surrounding bronchiectatic and fibrotic changes (Fig. 1). A diagnosis of multiple post-TB cavities with fungal ball (aspergilloma) was made. The patient underwent wedge resection of right upper lobe lesion. Tissue was sent for histopathology and culture. Histopathology revealed acute and chronic inflammation around the bronchioles, and hemorrhagic infarction; however, fungal hyphae were not visualized. Similarly $10 \%$ potassium hydroxide smear of tissue was negative for fungal hyphae. Culture after 3 days of incubation on Sabouraud dextrose agar (SDA) yielded white, cottony colonies that later turned gray with a pale reverse (Fig. 2). The growth was seen on all inoculated plates. Microscopic examination showed septate hyaline hyphae with conidia $9 \times 5 \mu \mathrm{m}$ in diameter borne terminally, singly, or in small groups on elongated simple or branched conidiophores or laterally on hyphae. The conidia were ovoid, with the larger end toward the apex, and appeared to be cut off at the base, with a distinct brown wall (Fig. 2b). The growth was not inhibited by cycloheximide. On the basis of macroscopic and microscopic features an identification of $S$. apiospermum was
Fig. 2 a Left front; flat, floccose colonies, a right reverse; pale yellow, $\mathbf{b}$ magnification $\times 40$, lactophenol cotton blue prepared slide from culture showing abundant oval conidia with scar at the base, larger end toward the apex, and appeared to be cut off at the base (arrow marked), c Voriconazole minimum inhibitory concentration by E test
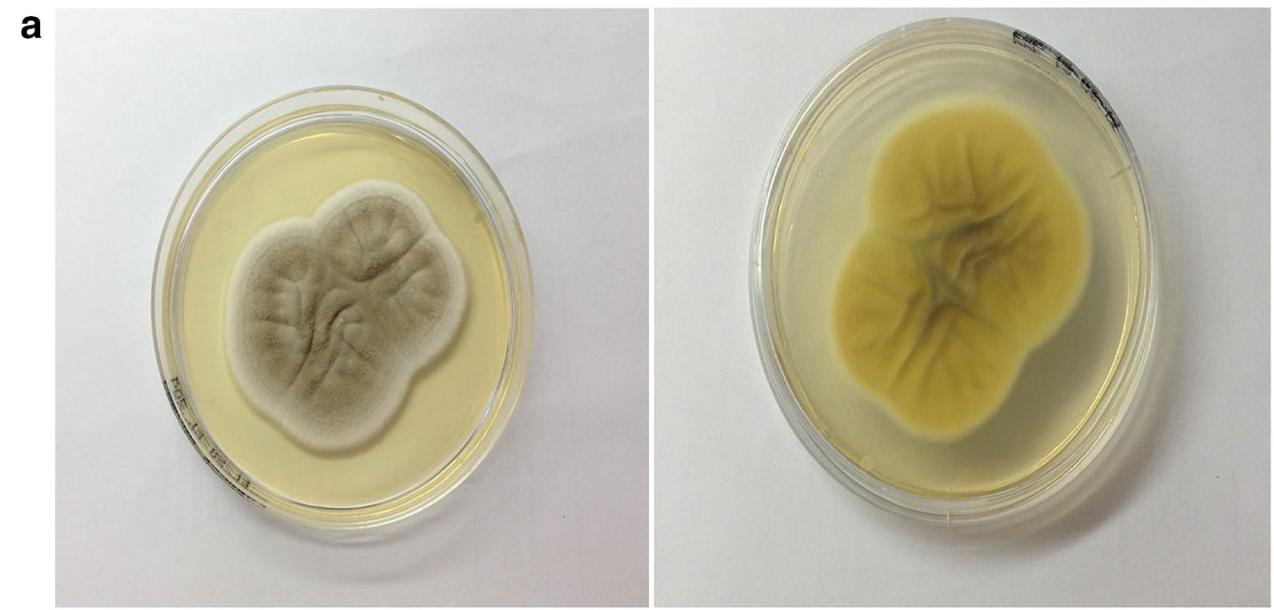

b
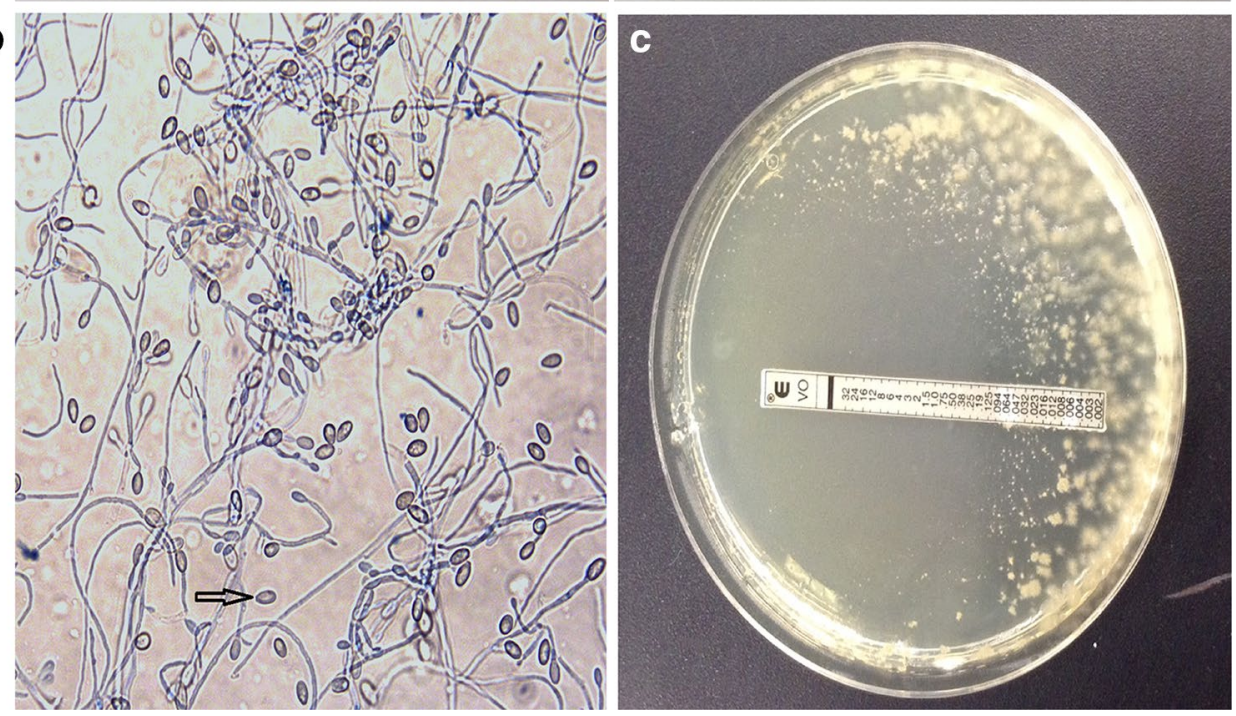
made [5]. The minimum inhibitory concentration (MICs) by $E$ test was $>32 \mu \mathrm{g} / \mathrm{ml}$ for amphotericin B, $>256 \mu \mathrm{g} / \mathrm{ml}$ for fluconazole, $>32 \mu \mathrm{g} / \mathrm{ml}$ for itraconazole and $0.06 \mu \mathrm{g} / \mathrm{ml}$ for voriconazole (Fig. 2c). Histopathology even on deeper sections and fungal stains, including periodic acid Schiff and Gomori methenamine silver, did not reveal any fungal elements. Original sample was reprocessed for fungus culture and it once again grew the same organism.

The patient was started on voriconazole, first two doses of the drug were administered at $400 \mathrm{mg} / 12 \mathrm{~h}$, and then the patient received maintenance doses of $200 \mathrm{mg} / 12 \mathrm{~h}(6 \mathrm{mg} /$ $\mathrm{kg} /$ day) for 4 months. Voriconazole plasma level could not be checked due to non-availability of diagnostic assay in Pakistan. He responded well to therapy. At 6-month follow-up he remained asymptomatic with chest X-ray improvement.

\section{Discussion and the literature review}

For the literature review we searched PubMed with the keywords "Pulmonary", "Lung", "Scedosporiosis", "Scedosporium", "Pseudallescheria", "boydii", "prolificans", "apiospermum" and "immunocompetent" in order to identify published cases of pulmonary scedosporiosis in an immunocompetent host. Seventeen cases were identified on PubMed (Table 1). Thus including current case, a total of 18 cases were analyzed for age and gender distribution, clinical symptoms and its duration, risk factor, treatment strategy and outcome. In case 6 no outcome was given. Therefore, case 6 was not analyzed in outcome analysis.

Gender and age analysis of cases (Table 1) showed maleto-female ratio of 11:7 with mean age distribution 53 years (range 27-72 years). Cough was the most common presentation (64\%) followed by hemoptysis (59\%) and fever $(53 \%)$. Duration of symptoms varied from acute presentation in near drowning cases to 10 years in patients with history of TB. Post-TB cavities $(44.5 \%, 8 / 18)$ were the most common risk factor to acquire pulmonary scedosporiosis. Surgery alone was used in 5/18 (28\%) and antifungal alone used in 7/18 (39\%). In 4/18 (22\%) cases, surgery along with antifungal therapy was used for treatment. Although overall case fatality rate was $35 \%(6 / 17), 50 \%(3 / 6)$ of cases that received antifungal therapy alone succumbed to death. All patients that underwent surgery alone were cured while $75 \%(3 / 4)$ cases were responded well to combination of surgery and antifungal therapy. Two cases did not receive any therapeutic intervention and both expired.

This study emphasizes the importance of including Scedosporium spp. in differential diagnosis of locally invasive lung infections as clinical, histopathological and radiographic examinations were unable to differentiate $\mathrm{Sce}$ dosporium from other filamentous fungi like Aspergillus.
This diagnostic confusion may delay the management of Scedosporium infection leading to poor outcomes. Castón et al. in a prospective cohort study found no difference between invasive pulmonary infection by Scedosporium apiospermum and invasive pulmonary aspergillosis on clinical grounds. However, a significant association was seen with prophylactic use of amphotericin B and development of invasive pulmonary scedosporiosis [19]. This relative resistance to amphotericin in Scedosporium spp. further highlights the significance of the definitive etiological characterization of Scedosporium through molecular diagnostics or culture.

Scrutinizing the cases regarding the gender distribution showed predominance of male $(62.5 \%)$ over female. Similarly in a review of 162 pulmonary and extra-pulmonary scedosporiosis in both immunocompetent and immunocompromised patients by Rodriguez-Tudela et al., there was a preponderance of infections in males by $63 \%$ [8].

Post-TB cavity formation was the most common risk factor for acquiring the pulmonary infections in most of the reported cases. Another review of cases of pulmonary scedosporiosis by Kantarcioglu et al. also highlights post-TB cavitation as a major risk factor [20]. As in our case study, high mortality rates with pulmonary scedosporiosis in immunocompetent patients have been reported in literature. Kantarcioglu et al. reported a mortality rate of 26.8 and $57.2 \%$ in patients with non-invasive and invasive infections, respectively [20].

On further review of the six cases with fatal outcome, two patients (Table 1; cases 7 and 9) did not receive any intervention, neither surgery nor antifungals. Three patients (Table 1; cases 8, 10 and 14) received antifungal therapy with ketoconazole, itraconazole and amphotericin B, respectively. The patient (Table 1; case 12) who received voriconazole for 1 month (dose not mentioned) deteriorated; lobectomy was performed but died within few hours of surgery. In cases with favorable outcome 8/11 (89\%) cases received surgical intervention and two of these patients (Table 1; cases 11 and 15) also received antifungal treatment initially. However due to poor response, the lesion was surgically excised. Three patients had received only antifungal therapy and in two of these patients (Table 1; cases 5 and 16) voriconazole dose was adjusted by monitoring plasma drug levels. The third patient (Table 1; case 17) received miconazole for 10 weeks, but due to its side effects it was replaced with ketoconazole. Chen et al. reported a case of disseminated Scedosporium infection in a patient with history of near drowning. The infection was cured by medical treatment with voriconazole without any surgical intervention [17]. However, in an analysis of 107 patients Troke et al. reported successful therapeutic response in only $57 \%$ of patients with scedosporiosis. In this study clinical outcome in pulmonary cases were not analyzed separately [7]. Rodriguez-Tudela 


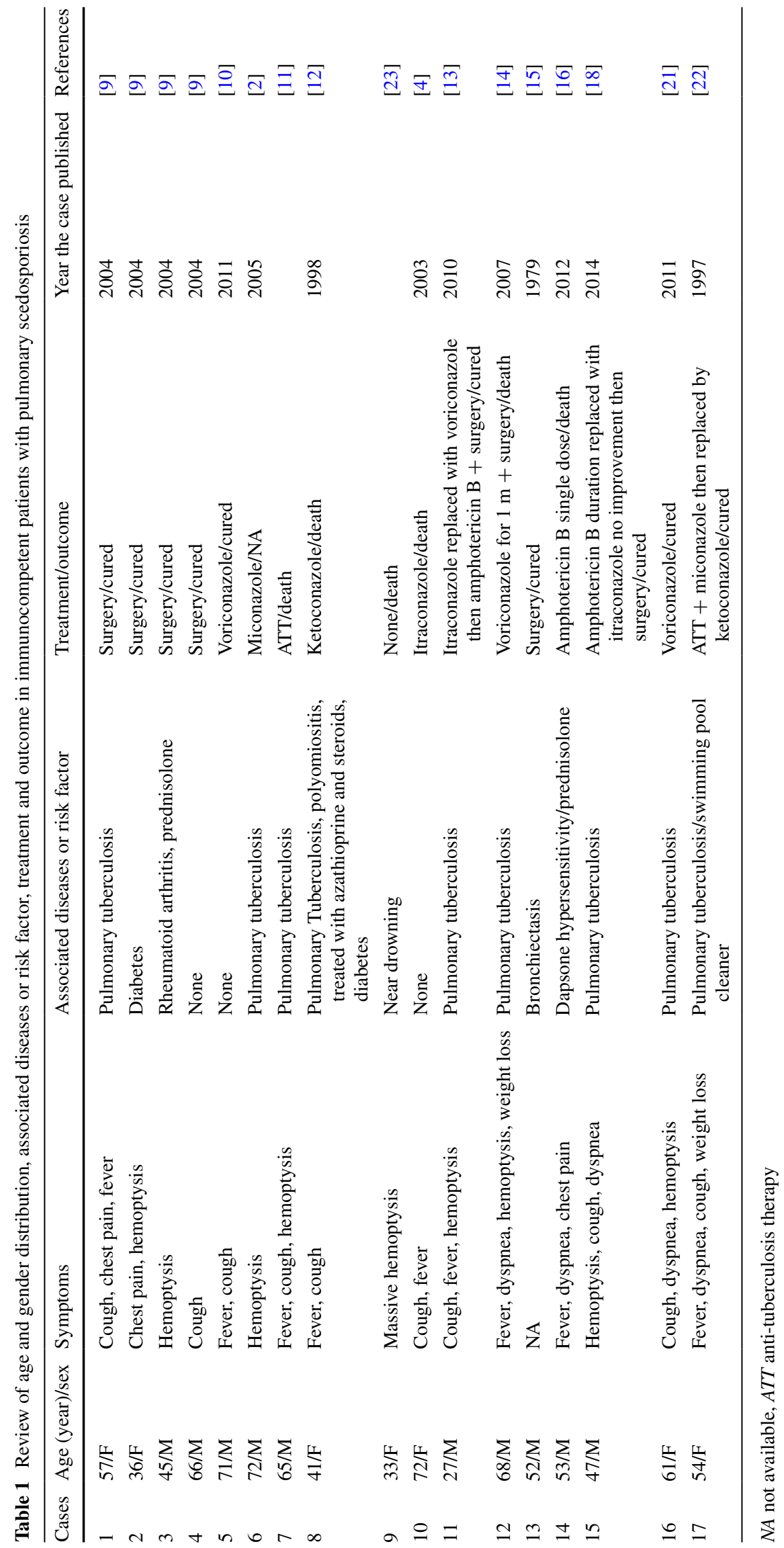


et al. showed that survival was independently associated with surgical excision [8]. Kantarcioglu et al. also reported that of 22/46 cases that survived underwent surgical excision as therapeutic intervention [20]. Outcome analysis in our study cases supports the surgical excision of the disease as a component of the standard of care.

In our case report, the isolated organism showed low MICs to voriconazole and high ones to fluconazole, itraconazole and amphotericin. Since no Clinical Laboratory Standards Institute breakpoints are available for susceptibility interpretation for Scedosporium spp., we were unable to categorize the strain as susceptible or resistant. This susceptibility pattern is supported by various in vitro studies that showed that Scedosporium has low MICs against voriconazole and high MICs against amphotericin B, itraconazole, fluconazole, ketoconazole and echinocandins $[6,7]$.

There was discrepancy with histopathological report of the lesion: no fungal elements were revealed despite examining deep sections with fungal stains. It was discovered that a very small sized tissue was sent for histopathology and which could very likely be non-representative of the entire sample. This highlights the importance of sending appropriate quantity and quality of specimens sample for laboratory investigations. The likelihood of contamination of the specimen in the laboratory was eliminated by two means: growth of the same organism appeared on all culture plates and reprocessing of culture again yielded the same organism.

We were unable to confirm the identification of the isolate by DNA sequencing due to limited resources. However, Scedosporiuum apiospermum is one of the more commonly seen fungi in the clinical laboratory, its phenotypic identification by our laboratory can be relied upon as our laboratory regularly participates in external quality assurance by College of American Pathologists and has consistently shown satisfactory results.

\section{Conclusion}

Every fungal ball in preformed lung cavities is not an aspergilloma. Fungal cultures or molecular diagnostic tools are necessary for definite diagnosis and appropriate therapeutic measures.

Acknowledgments Antifungal susceptibility testing is supported through grants from the Higher Education Commission, Pakistan, and United States Agency for international Development. We are thankful to Dr. Joveria Farooqi for proof reading of the manuscript for English language and grammar.

\section{Compliance with ethical standards}

Conflict of interest The authors declare no conflict of interest.

\section{References}

1. Cortez KJ, Roilides E, Quiroz-Telles F, Meletiadis J, Antachopoulos. Infections caused by Scedosporium spp. Clin Microbiol Rev. 2008;21:157-97.

2. Koga T, Kitajima T, Tanaka R, Hirokawa M, Ichiki M, Rikimaru $\mathrm{T}$, et al. Chronic pulmonary scedosporiosis simulating aspergillosis. Respirology. 2005;10:682-4.

3. Al Refaï M, Duhamel C, Le Rochais JP, Icard P. Lung scedosporiosis: a differential diagnosis of aspergillosis. Eur J Cardiothorac Surg. 2002;21:938-9.

4. Horré R, Jovanić B, Marklein G, Schumacher G, Friedrichs $\mathrm{N}$, Neuhaus T, de Hoog GS. Fatal pulmonary scedosporiosis. Mycoses. 2003;46:418-21.

5. Larone DH. Medically important fungi. 4th ed. Washington, DC: ASM Press; 2002.

6. Girmenia C, Luzi G, Monaco M, Martino P. Use of voriconazole in treatment of Scedosporium apiospermum infection: case report. J Clin Microbiol. 1998;36:1436-8.

7. Troke P, Aguirrebengoa K, Arteaga C, Ellis D, Heath CH, Lutsar I, et al. Treatment of scedosporiosis with voriconazole: clinical experience with 107 patients. Antimicrob Agents Chemother. 2008;52:1743-50.

8. Rodriguez-Tudela JL, Berenguer J, Guarro J, Kantarcioglu AS, Horre R, de Hoog GS, et al. Epidemiology and outcome of Scedosporium prolificans infection, a review of 162 cases. Med Mycol. 2009;47:359-70.

9. Severo LC, Oliveira F de M, Irion K. Respiratory tract intracavitary colonization due to Scedosporium apiospermum: report of four cases. Rev Inst Med Trop Sao Paulo. 2004;46:43-6.

10. Ogata R, Hagiwara E, Shiihara J, Ogura T, Takahashi H, Kamei $\mathrm{K}$. A case of lung scedosporiosis successfully treated with monitoring of plasma voriconazole concentration level. Nihon Kokyuki Gakkai Zasshi. 2011;49:388-92.

11. Severo LC, Londero AT, Picon PD, Rizzon CFC, Tarasconi JC. Petriellidium boydii fungus ball in a patient with active tuberculosis. Mycopathologia. 1982;77:15-7.

12. Severo LC, Porto NS, Londero AT. Pulmonary scedosporiosis. Rev Inst Med Trop Sao Paulo. 1998;40:241-3.

13. Soman R, Mahashur AA, Naphade D, Rodrigues C, Bhaduri A, et al. Pseudallescheria boydii lung infection in an immunocompetent adult, difficulties in diagnosis and management. J Assoc Physicians India. 2010;58:633-4.

14. Abgrall S, Pizzocolo C, Bouges-Michel C, Martinod E, Martin A, Brauner M, et al. Scedosporium apiospermum lung infection with fatal subsequent postoperative outcome in an immunocompetent host. Clin Infect Dis. 2007;45:524-5.

15. Deloach ED, DiBenedetto RJ, Hitch WS, Russell P. Pulmonary infection with Petriellidium boydii. South Med J. 1979;72:479-81.

16. Ceccarelli L, Calisti G, Delle Rose D, Ricciardi A, Maffongelli G, Sordillo P, et al. Dapsone hypersensitivity syndrome complicated by Scedosporium apiospermum pneumonia in an immunocompetent patient. Infection. 2012;40:459-62.

17. Chen TC, Ho MW, Chien WC, Lin HH. Disseminated Scedosporium apiospermum infection in a near-drowning patient. $\mathrm{J}$ Formos Med Assoc. 2015. doi:10.1016/j.jfma.2015.02.007.

18. Agatha D, Krishnan KU, Dillirani VA, Selvi R. Invasive lung infection by Scedosporium apiospermum in an immunocompetent individual. Indian J Pathol Microbiol. 2014;57:635-7.

19. Castón JJ, Linares MJ, Rivero A, Casal M, Torre-Cisneros J. Clinical differences between invasive pulmonary infection by Scedosporium apiospermum and invasive pulmonary aspergillosis. Mycoses. 2011;54:e468-73. 
20. Kantarcioglu AS, de Hoog GS, Guarro J. Clinical characteristic and epidemiology of pulmonary pseudallescheriasis. Rev Iberoam Micol. 2012;29:1-13.

21. Durand CM, Durand DJ, Lee R, Ray SC, Neofytos D. A 61 yearold female with a prior history of tuberculosis presenting with hemoptysis. Clin Infect Dis. 2011;52:910 (957-9).
22. Tekavec J, Mlinarić-Missoni E, Babic-Vazic V. Pulmonary tuberculosis associated with invasive pseudallescheriasis. Chest. 1997;111:508-11.

23. Nakadate T, Nakamura Y, Yamauchii K, Endo S. Two cases of severe pneumonia after the 2011 Great East Japan Earthquake. Western Pac Surveill Response J. 2012;3:67-70. 